\title{
Fibrodysplasia ossificans progressiva - can we diagnose it right at the outset?
}

\author{
Amit Sharma ${ }^{1}$, Dhruv Maini ${ }^{2}$, Gaurang Agarwal ${ }^{1}$, Parul Sharma ${ }^{3}$, Lalit Maini ${ }^{1}$ \\ ${ }^{1}$ Department of Orthopaedics, Maulana Azad Medical College, Delhi; ${ }^{2}$ Gajra Raja Medical College, Gwalior, India, \\ ${ }^{3}$ Department of Physiotherapy, Jamia Milia Islamia, Delhi.E-mail: dramite31@gmail.com \\ Received: 30th June 2018, Revised: 17th November 2018, Accepted: 24th December 2018
}

SUMMARY: Sharma A, Maini D, Agarwal G, Sharma P, Maini L. Fibrodysplasia ossificans progressiva - can we diagnose it right at the outset? Turk J Pediatr 2019; 61: 958-962.

Fibrodysplasia ossificans progressiva (FOP) is a rare autosomal dominant disorder with no definitive treatment options available yet, except for physiotherapy and bisphosphonates. Due to its initial presentation with multiple lumps in the body, it is often misdiagnosed as a benign tumour most commonly being an osteochondroma or Olliers syndrome. Delay in diagnosis not only delays the management but can also expose the patient to unnecessary interventions. Moreover, earlier diagnosis can also make the patient aware of the precautions to be taken. So our remark is "can we diagnose this disease right at the outset"? We present a case of a 10 year old boy, who had all the classical features of FOP yet was misdiagnosed. Therefore, classical hallmark features of this disease are highlighted in this case report which can be picked up easily by any clinician to reach to a definitive diagnosis as early as possible avoiding unnecessary iatrogenic insult.

Key words: bisphosphonates, fibrodysplasia ossificans progressiva, heterotopic, ossification, osteochondroma.

Fibrodysplasia ossificans progressiva (FOP) is a rare autosomal dominant disorder which is also known as fibrodysplasia ossificans progressiva, stiff man syndrome, usually presents as ectopic ossification of ligaments, tendons and within the joints. Disease in itself is very crippling, making patient wheel chairbound in the 2 nd decade of life, with a reduced life span, too. This disease is extremely rare with less than 1000 cases reported yet, with an estimated incidence rate of one in 2 million. ${ }^{1,2}$ It is a progressive disorder with male preponderance, which usually presents in the first decade of life. Features like congenital malformation of great, bilateral hallux valgus, monophalangism, and progressive heterotopic ossification are the hallmark characteristics of FOP. Ossification usually involves muscle, tendons and ligaments of axial skeleton. In the first decade of life, the disease usually manifests as small lumps which are usually present at the back around the neck and shoulder, gradually involving lower back, hip and other joints progressing caudally. These lumps are basically soft tissue swelling formed secondary to heterotopic ossification. ${ }^{3}$ Unfortunately, there are still not many treatment options available for FOP but physiotherapy and bisphosphonates. ${ }^{4}$ Bisphophonates and ascorbic acids are the only mainstay of treatment currently, which seems to act by stabilizing the disease and slowing down the process of ossification. Hence, the only way to provide some sort of benefit to such patients is by an earlier diagnosis at the outset. The earlier we start physiotherapy and increase patient awareness, the slower is the progression of disease. ${ }^{4}$ Hence, we report on a 10 -year-old boy who presented with the rapidly progressive disease after a history of trauma, with hallmark diagnostic clinical features not to be missed by a clinician. 


\section{Case Report}

In January 2018, a 10-year-old boy presented in our outpatient department with a history of restricted bilateral shoulder movements and multiple lumps over the back for the last 6 months. Restriction of movement of bilateral shoulder had increased to such an extent that the patient was not able to feed himself for the previous 2 months. Patient also gave history that onset of lumps was preceded by an incidence of trauma to back and shoulder, one year back while playing, which did not cause any fracture. No history of any other systemic disorder was taken. On detailed examination, multiple lumps were found on the mid-back adjacent to bilateral scapulae (Fig. 1). Shoulder abduction, bilaterally, was only 30 degrees with completely restricted rotation and flexion. On examination of other joints, bilateral elbow were found to have flexion deformity of 15 degree with further flexion possible till 100 degree suggestive of terminal restriction of motion, and stiff neck with inability of child to touch the chin to chest was also noticed. Patient also had a lump in the proximal tibia located postero-medially looking like osteochondroma.

Hallmark diagnostic features for FOP were also present, which included bilateral monophalangeal great toes, short $1^{\text {st }}$ metatarsals with bilateral halluces valgus and short $1^{\text {st }}$ metacarpals (Figs. 2-3).

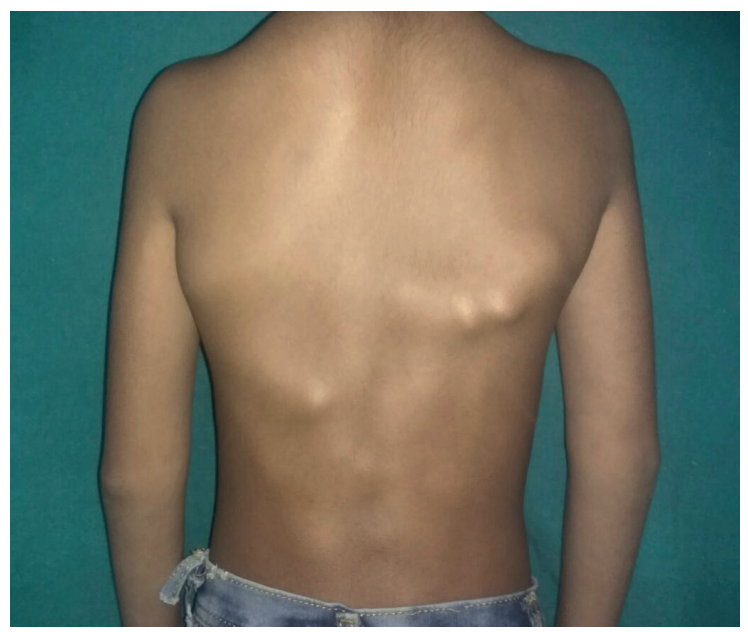

Fig. 1. Image depicting multiple lumps on the back of the patient, mimicking multiple osteochondromas.

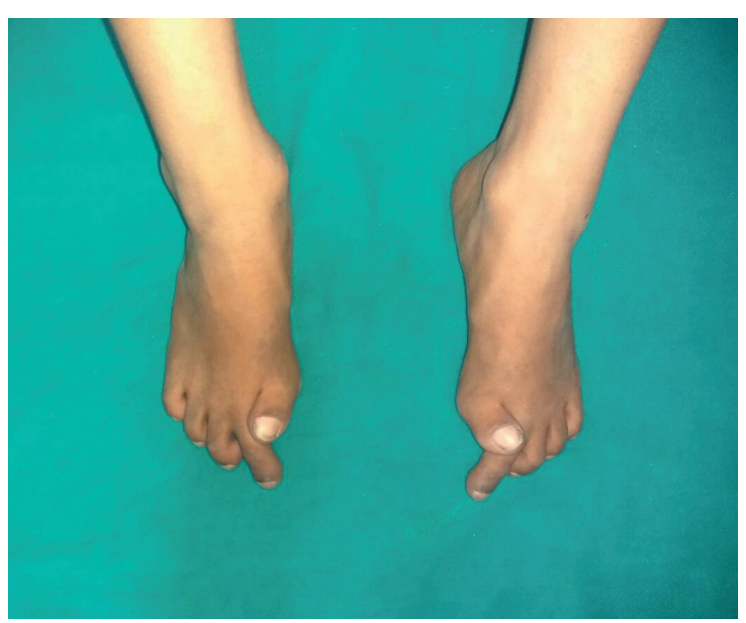

Fig. 2. Hallmark diagnostic sign of fibrodysplasia ossificans progressiva i.e. Short 1st halluces with bilateral valgus deformities.

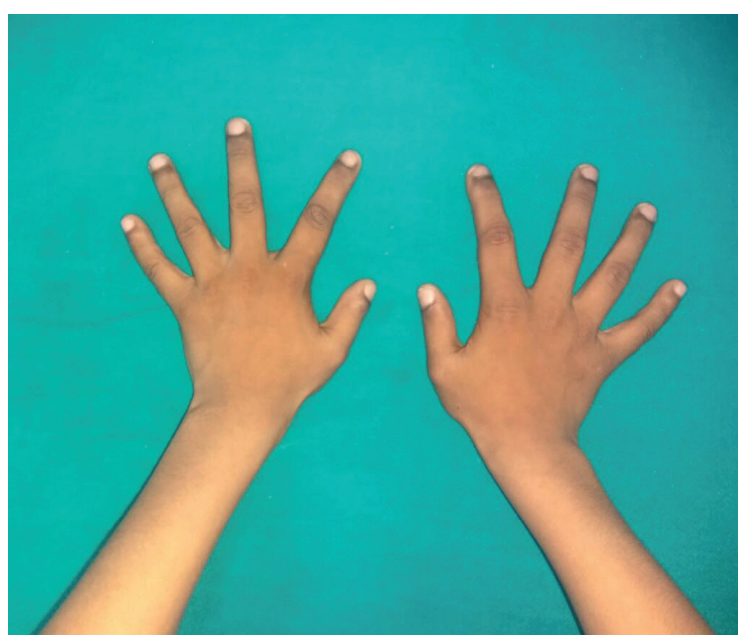

Fig. 3. Image of bilateral hands depicting bilateral short 1st metacarpals.

Routine laboratory investigations were conducted including complete blood count, creatine kinase, LDH, liver function tests and renal function tests; all which were found to be normal. Spine X-ray showed ossification in muscles and ligaments with confirmation of monophalangeal great toe and short $1^{\text {st }}$ metacarpals and metatarsals (Figs. 4-7).

Even with the entire set of diagnostic findings, the patient was misdiagnosed as Olliers disease and was treated accordingly at a peripheral centre. Moreover, after the incidence of trauma, patient was advised to receive massage therapy which further aggravated the 


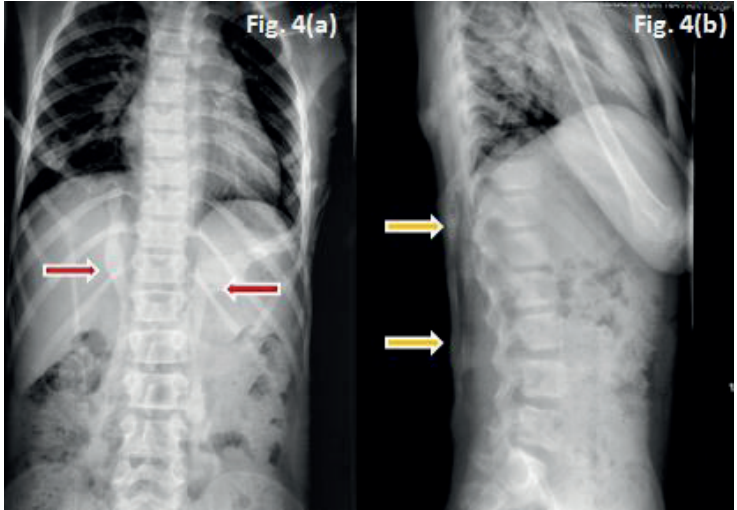

Fig. 4. X-ray views of lumbar spines; anteroposterior (left panel) and lateral (right panel), depicting ossification of back muscles, ligaments and subcutaneous tissue (arrow heads).

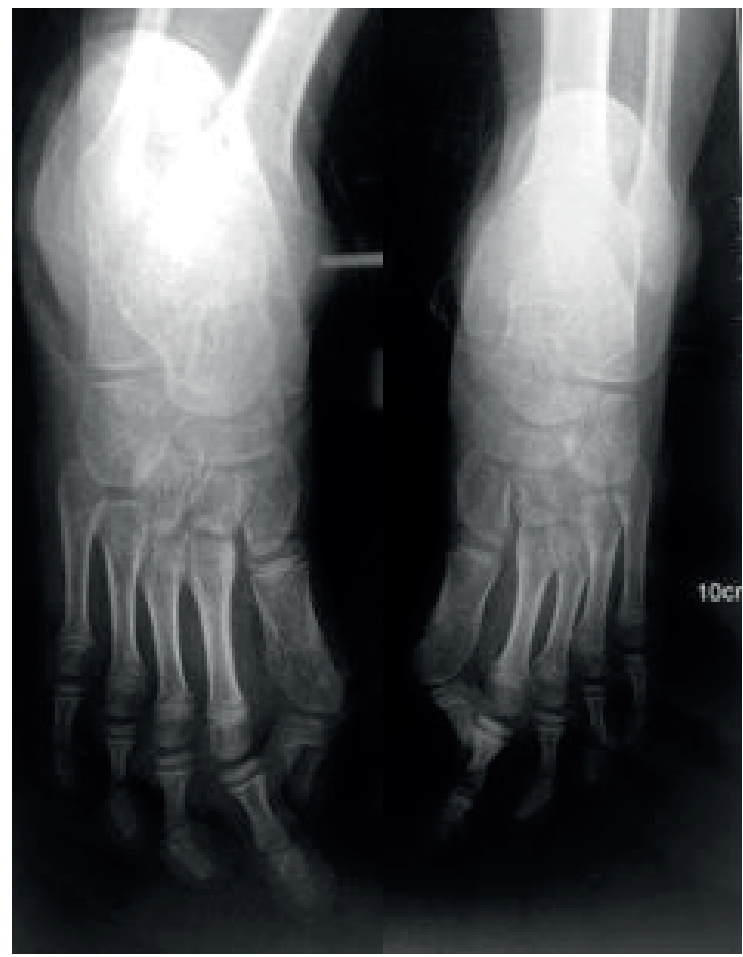

Fig. 5. X-ray views of bilateral feet showing monophalangeal great toes with short $1^{\text {st }}$ metatarsals and bilateral valgus deformities.

disease progression. Thus, the fact needs to be highlighted and emphasized that there is a need of diagnosing such patients at the outset, so that we can counsel the patient accordingly and start an early rehabilitation program.

Informed consent was obtained from patient and his family members, regarding participation as case report.

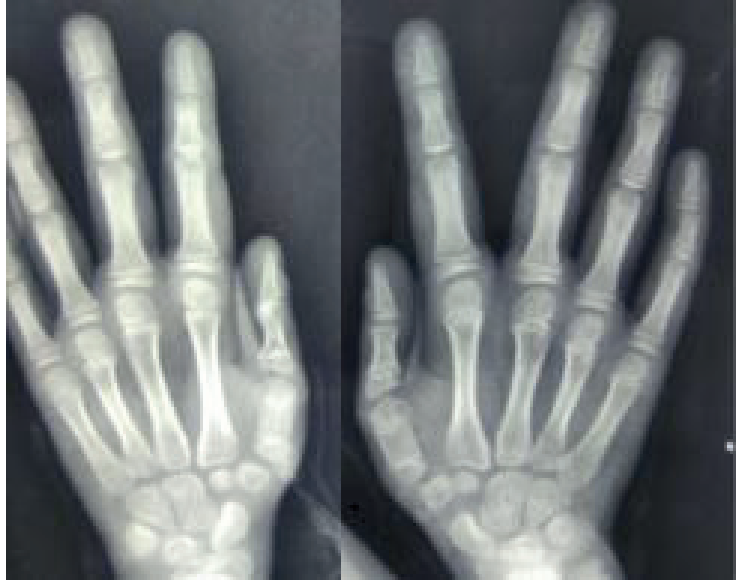

Fig. 6. X-ray views of bilateral hands showing short $1^{\text {st }}$ metacarpals bilaterally (sent priginal pic of both hands AP view).

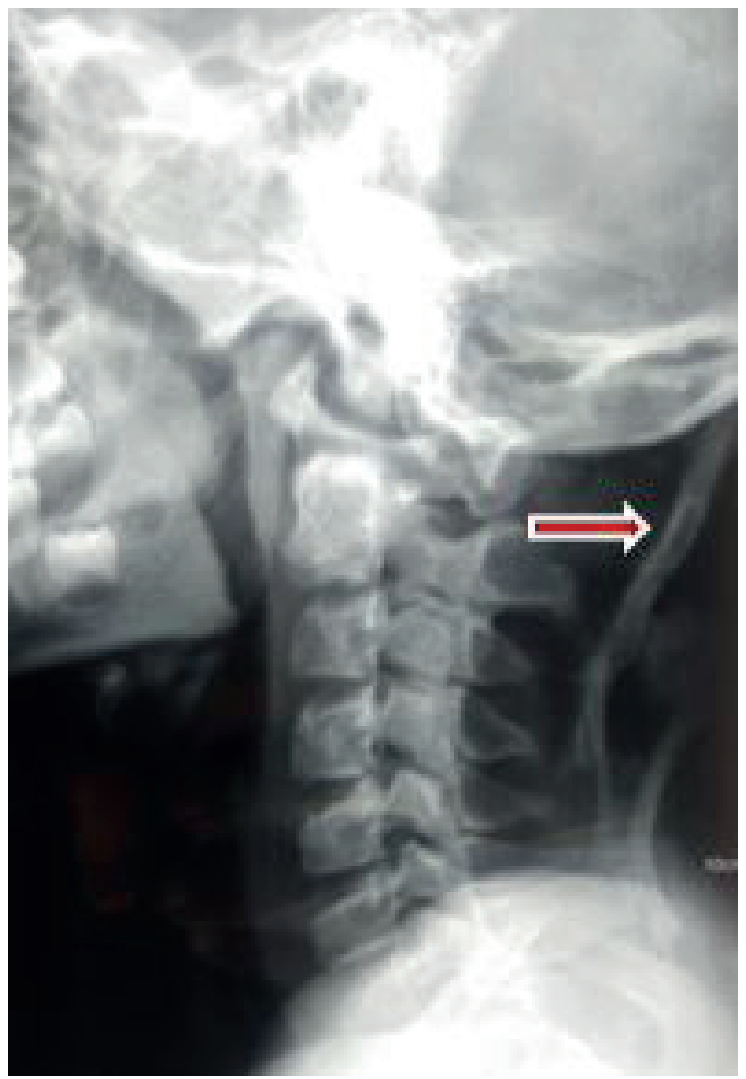

Fig. 7. X-ray views of cervical spines. Lateral view depicting ossification (arrow) of neck muscles, ligaments and subcutaneous tissue.

\section{Discussion}

Corollary of FOP is that, though being so severe, it often goes unnoticed till late and may be diagnosed with some benign lesions like multiple osteochondroma in the initial 
phase of the disease. The cytoplasmic domain of the activin receptor IA/activin like kinase 2 (ACVR1/ALK2), a bone morphogenetic protein (BMP) type I receptor leading to dysregulated BMP signalling are the major genes involved in the pathogenesis of FOP. The disease follows an autosomal dominant inheritance pattern. ${ }^{5}$

The disease progresses rapidly with each attack, which can be easily induced by any soft tissue injury, intramuscular injection, muscular stretching, biopsy or any other local trauma. The same has been observed in our case in which the patient gave a history of trauma while playing. Gradually the disease progresses to such an extent that the patient usually becomes wheel chair-bound by the age of 20 and the life expectancy is also usually restricted to $3^{\text {rd }}-4^{\text {th }}$ decade. ${ }^{6,7}$ The cause of death is usually involvement of chest causing respiratory insufficiency and right-sided heart failure. Cardiac muscles, extraocular muscles, tongue and diaphragm are usually spared suggesting the role of physiotherapy in initial phase to curtail the progress of disease. ${ }^{8}$

Early diagnosis of the condition minimizes unnecessary invasive investigations like a biopsy, which makes the situation even worse, as cancer is the most common differential diagnosis worldwide almost $87 \%$ of the time. ${ }^{9}$ Moreover it will make the family aware of the disease in order to avoid any local trauma, including even injections. Hence, diagnostic skeletal manifestation should be picked up by a clinician as early as possible so that the patient can be made wise to avoid any local traumatic insults which can flare up the disease, and patient can be educated about the role of mild physiotherapy at the early age which may delay the progress of the disease. These diagnostic skeletal manifestations include ectopic calcifications, short hallux with either monophalangism or synphalangism, bilateral halluces valgus (75-90\%), short 1 st thumbs secondary to short first metacarpals, restricted range of motion of shoulders, kyphoscoliosis with lumps over the back, intellectual disability, alopecia and cardiac conduction defect are other rare finding. ${ }^{10,11} \mathrm{It}$ is important to remember that these features may not always accompany the condition, and besides, there are many genetic conditions in differential diagnosis with overlapping skeletal features.

The present case had 5 diagnostic clinical features, including characteristic monophalangeal great toes, bilateral halluces valgus, yet the disease was not diagnosed by the age of 10 years. Kittermann et al. ${ }^{9}$ also discussed in their study of 138 patients about the median age of diagnosis of FOP, which they found to be delayed by 4.1 years. Moreover a patient usually visits 6 physicians on an average before reaching the correct diagnosis. ${ }^{9}$ At our centre diagnosis was based on the characteristic clinical and radiological findings, amongst which most important were soft tissue ossification and lumps associated with bilateral shortening of big toes with hallux valgus, which is seen in over $95 \%$ of the patients. Despite the known fact of a poor prognosis, the patient has now been put on a rehabilitation protocol with bisphosphonates with oral ascorbic acid. Other available treatment options in such a patient are avoidance of trauma, passive physiotherapy of all joints, chest physiotherapy, and prevention of chest infection, regular audiometric and cardiac analysis. Steroids are used only to reduce the inflammation during the onset of a flare up within 24 hours for 4 days. ${ }^{12}$ No other medical drug therapy has yet to be found successful. However some newer therapies like rosiglitazone, antiangiogenic drugs like squalamine, thalidomide, COX-2 inhibitors, BMP4 antagonist and noggin and gremlin gene therapy are under trial.

Hereby, we conclude that bilateral shortening of great toes may be clinical hallmarks of this rare disorder, and although there are no definitive treatment options available yet, early diagnosis may prevent further iatrogenic insults and promote family counselling for prevention of further trauma.

\section{REFERENCES}

1. Whyte MP. Heritable metabolic and dysplastic bone diseases. Endocrinol Metab Clin North Am 1990; 19: 133-173.

2. Connor JM, Evans DA. Genetic aspects of fibrodysplasia ossificans progressiva. J Med Genet 1982; 19: 35-39. 
3. Pignolo RJ, Suda RK, Kaplan FS. The fibrodysplasia ossificans progressiva lesion. Clin Rev Bone Miner Metabol 2005; 3: 195-200.

4. Palhares DB, Leme LM. A perspective on the control of myositis ossificans progressiva. J Pediatr (Rio J) 2001; 77: 431-434.

5. Shore EM, Xu M, Feldman GJ, et al. A recurrent mutation in the BMP type I receptor ACVR1 causes inherited and sporadic fibrodysplasia ossificans progressiva. Nat Genet 2006; 38: 525-527.

6. Cohen RB, Hahn GV, Tabas JA, et al. The natural history of heterotopic ossification in patients who have fibrodysplasia ossificans progressiva. A study of forty-four patients. J Bone Joint Surg Am 1993; 75: 215-219.

7. Kaplan FS, Glaser DL, Shore EM, et al. The phenotype of fibrodysplasia ossificans progressiva. Clin Rev Bone Miner Metab 2005; 3: 183-188.
8. Kaplan FS, Glaser DL. Thoracic insufficiency syndrome in patients with fibrodysplasia ossificans progressiva. Clin Rev Bone Miner Metab 2005; 3: 213-216.

9. Kitterman JA, Kantanie S, Rocke DM, Kaplan FS Iatrogenic harm caused by diagnostic errors in fibrodysplasia ossificans progressiva. Pediatrics 2005; 116: e654-e661.

10. Tederko P, Krasuski M, Kiwerski J, Barcinska I. The importance of verifying diagnosis in patients with spinal cord injury hospitalized in a rehabilitation department. Ortho Traumatol Rehabil 2005; 7: 365373.

11. Kapoor R, Gadre PM, Mattoo P, Agrawal R. Fibrodysplasia ossificans progressiva. Indian Pediatr 1998; 35: 786-788.

12. Oshin AO, Stewart MC. The role of bone morphogenetic proteins in articular cartilage development, homeostasis and repair. Vet Comp Orthop Traumatol 2007; 20: 151-158. 